\title{
RESOLUTION OF NULL FIBER AND CONORMAL BUNDLES ON THE LAGRAGIAN GRASSMANNIAN
}

\author{
KYO NISHIYAMA \\ Dedicated to Professor Noriaki Kawanaka on his 60th anniversary
}

\begin{abstract}
We study the null fiber of a moment map related to dual pairs. We construct an equivariant resolution of singularities of the null fiber, and get conormal bundles

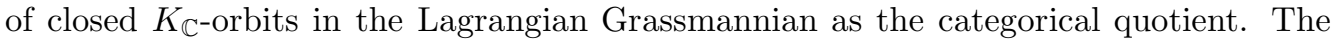
conormal bundles thus obtained turn out to be a resolution of singularities of the closure

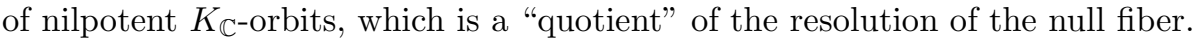

\section{CONTEnTs}

Introduction

Notation

1. Contraction map and its null fiber

2. Resolution of null fibers

2.1. Stable range case $\left(n \geq m_{1}+m_{2}\right)$

2.2. Outside of the stable range $\left(n<m_{1}+m_{2}\right)$

2.3. Reduction to the stable range lift 7

3. Dual pair and moment maps 9

4. Intersection of Lagrangian flag varieties 11

5. Case of $\mathrm{Sp}_{2 n}(\mathbb{R}) \times \mathrm{O}_{n, n}(\mathbb{R}) \quad 13$

6. Case of $\mathrm{O}_{2 n}^{*} \times \mathrm{Sp}_{n, n} \quad 15$

References

\section{INTRODUCTION}

Let $\mathrm{M}_{m, n}=\mathrm{M}_{m, n}(\mathbb{C})$ be the space of $m \times n$-matrices with coefficients in $\mathbb{C}$. Then the map $\psi: \mathrm{M}_{m_{1}, n} \times \mathrm{M}_{n, m_{2}} \rightarrow \mathrm{M}_{m_{1}, m_{2}}$ defined by $\psi(A, B)=A B$ is called a contraction map. There are many interpretations of this simple map. Let us explain some of them.

2000 Mathematics Subject Classification. Primary 14M15, 14E15; Secondary 14L30, 22E47, 20 G05.

Key words and phrases. resolution, null fiber, conormal bundle, Lagrangian Grassmannian, flag variety, dual pair, nilpotent orbit. 
The general linear group $K=\mathrm{GL}_{n}(\mathbb{C})$ acts on the space $W=\mathrm{M}_{m_{1}, n} \times \mathrm{M}_{n, m_{2}}$ naturally by $g \cdot(A, B)=\left(A g, g^{-1} B\right)(g \in K)$. Then clearly the contraction map is invariant under the action of $K$. It turns out that $\psi$ is the affine quotient map onto its image $W / / K=\operatorname{Spec}\left(\mathbb{C}[W]^{K}\right)$, which is the determinantal variety of $\operatorname{rank} r=\min \left\{m_{1}, m_{2}, n\right\}$ (see Equation (1.2)).

The fiber $\mathfrak{N}_{K}(W)=\psi^{-1}(0)$ of the quotient map is called the null fiber or null cone. In the invariant theory, the study of the null fiber is important since it is the "worst" fiber of the quotient map. For example, if it has a finite number of orbits, then the other fibers also have a finite number of orbits. If it is reduced and irreducible, then so are the other fibers. In the following, we will clarify the structure of the null fiber $\mathfrak{N}_{K}(W)$ to give the orbital decomposition, the irreducible decomposition (as an algebraic variety), $K$-module structure of the function ring, etc., though most of these results seem to be classical.

Our main interest in this paper is in the construction of resolution of singularities for each irreducible component of $\mathfrak{N}_{K}(W)$, which is $K$-equivariant. To state our main results on the resolutions, we need to explain another interpretation of the contraction map.

Let us consider a dual pair $\left(\mathrm{Sp}_{2 n}(\mathbb{R}), \mathrm{O}_{m_{1}, m_{2}}(\mathbb{R})\right)$ in the large symplectic group $\mathrm{Sp}_{2 N}(\mathbb{R})$ where $N=n\left(m_{1}+m_{2}\right)$. Then the vector space $W$ above can be considered as a Lagrangian subspace in the symplectic space $\mathbb{C}^{N}=\mathrm{M}_{2 n, m_{1}+m_{2}}$, and $\psi$ is a moment map associated to the dual pair.

Note that $K=\mathrm{GL}_{n}(\mathbb{C})$ is the complexification of a maximal compact subgroup of $\mathrm{Sp}_{2 n}(\mathbb{R})$, and if we take a Cartan decomposition of $\mathfrak{o}_{m_{1}, m_{2}}(\mathbb{R})=\mathfrak{k}_{\mathbb{R}}^{\prime} \oplus \mathfrak{p}_{\mathbb{R}}^{\prime}$, the target space $\mathrm{M}_{m_{1}, m_{2}}$ is isomorphic to the complexification $\mathfrak{p}^{\prime}=\mathfrak{p}_{\mathbb{R}}^{\prime} \otimes_{\mathbb{R}} \mathbb{C}$ of the Cartan space.

In completely analogous way, there also exists another moment map $\varphi: W \rightarrow \mathfrak{p}=$ $\operatorname{Sym}_{n}(\mathbb{C}) \oplus \operatorname{Sym}_{n}(\mathbb{C})^{*}$, where $\mathfrak{p}$ denotes (the complexification of) a Cartan space for $\mathrm{Sp}_{2 n}(\mathbb{R})$. We can lift nilpotent orbits in $\mathfrak{p}^{\prime}$ to those in $\mathfrak{p}$ using these moment maps ([Nis05], [NOZ06], DKP02, DKP05]; see §3 for details). In this terminology, the quotient of the null fiber $\mathfrak{N}_{K}(W)$ by the action of $K^{\prime}=\mathrm{O}_{m_{1}}(\mathbb{C}) \times \mathrm{O}_{m_{2}}(\mathbb{C})$ (the complexification of a maximal compact subgroup of $\left.\mathrm{O}_{m_{1}, m_{2}}(\mathbb{R})\right)$ gives a collection of nilpotent orbits for the symmetric pair $\left(\operatorname{Sp}_{2 n}(\mathbb{R}), \mathrm{U}_{n}(\mathbb{C})\right)$ which is usually denoted $(+-)^{p}(-+)^{q}(+)^{n-(p+q)}(-)^{n-(p+q)}$ in terms of signed Young diagrams. Let us denote this orbit by $\mathbb{O}_{p, q}$.

To state our main results, we assume $n=m_{1}=m_{2}$, i.e., we consider the equal rank case. The following theorems are summary of main results in this article.

Theorem A. Assume $n=m_{1}=m_{2}$, and put $K=\mathrm{GL}_{n}(\mathbb{C})$ and $K^{\prime}=\mathrm{O}_{n}(\mathbb{C}) \times \mathrm{O}_{n}(\mathbb{C})$. Then the null fiber has an irreducible decomposition

$$
\mathfrak{N}_{K}(W)=\bigcup_{p+q=n} \mathfrak{N}_{p, q}
$$

with $(n+1)$ irreducible components. For each component $\mathfrak{N}_{p, q}(p+q=n)$, there exists a $K$-equivariant resolution of singularities, which is denoted by $\nu: \widetilde{\mathfrak{N}}_{p, q} \rightarrow \mathfrak{N}_{p, q}$.

The resolution $\widetilde{\mathfrak{N}}_{p, q}$ is realized as a vector bundle over Grassmannian variety $\operatorname{Grass}_{p}\left(\mathbb{C}^{n}\right)$ of $p$-dimensional subspaces in $\mathbb{C}^{n}$ (see $\$ 2.2$ and Proposition 2.2 for details). Let $\mathfrak{X}^{-}$be the 
Lagrangian Grassmannian of all the Lagrangian subspaces in $\mathbb{C}^{2 n}$, which is isomorphic to a partial flag variety of $\mathrm{Sp}_{2 n}(\mathbb{C})$ with respect to the Siegel parabolic subgroup. Then $K$ naturally acts on $\mathfrak{X}^{-}$, and it has exactly $(n+1)$ closed orbits $\mathcal{Z}_{p, q}(p+q=n)$ and $\mathcal{Z}_{p, q}$ is isomorphic to $\operatorname{Grass}_{p}\left(\mathbb{C}^{n}\right)$ (Lemma 4.2).

Theorem B (Theorem 5.1). With the same assumption above, the categorical quotient $\widetilde{\mathfrak{N}}_{p, q} / / K^{\prime}$ of the resolution exists, and it is isomorphic to the conormal bundle $T_{\mathcal{Z}_{p, q}}^{*} \mathfrak{X}^{-}$ over the closed $K$-orbit $\mathcal{Z}_{p, q}$ in the Lagrangian flag variety $\mathfrak{X}^{-}$.

There is a natural moment map $\mu$ from the cotangent bundle $T^{*} \mathfrak{X}^{-}$to the nilpotent variety. If we restrict $\mu$ to the conormal bundle $T_{\mathcal{Z}_{p, q}}^{*} \mathfrak{X}^{-}$, it is known to be the closure of a nilpotent $K$-orbit.

Theorem $\mathbf{C}$ (Theorem 5.2). The image of the moment map $\mu: T_{\mathcal{Z}_{p, q}}^{*} \mathfrak{X}^{-} \rightarrow \mathfrak{p}$ coincides with the closure of the nilpotent orbit $\overline{\mathbb{O}}_{p, q} \simeq \mathfrak{N}_{p, q} / / K^{\prime}$. Thus we have the following commutative diagram.

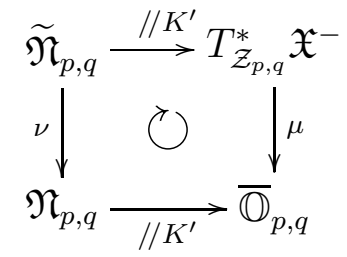

If $n$ is even, there is an analogue of the above theorems for the dual pair $\left(\mathrm{O}_{2 n}^{*}, \mathrm{Sp}_{n . n}\right)$. See $\$ 6$ for details.

The results of this article are purely geometric. However, we believe these geometrical properties of the null fiber reflect to the analysis of degenerate principal series of $\mathrm{Sp}_{2 n}(\mathbb{R})$ and the theta liftings from $\mathrm{O}_{n, n}(\mathbb{R})$ to $\mathrm{Sp}_{2 n}(\mathbb{R})$. The representation theoretic interpretations of the geometry of the null fiber will be treated in the forthcoming paper.

Acknowledgment. The author thanks to Soo Teck Lee and Peter Trapa for useful discussions.

\section{NotATiON}

Let $\mathcal{P}_{n}$ be the set of all the partitions $\alpha=\left(\alpha_{1}, \alpha_{2}, \ldots, \alpha_{n}\right), \alpha_{1} \geq \alpha_{2} \geq \cdots \geq \alpha_{n} \geq 0$ with length less than or equal to $n$. Here, the length of the partition $\alpha$, denoted by $\ell(\alpha)$, is defined by the biggest number $l$ such that $\alpha_{l}>0=\alpha_{l+1}$. We naturally identify $\mathcal{P}_{n}$ with the subset of $\mathcal{P}_{n+1}$ by extending the sequence by adding 0 .

For $\lambda \in \mathbb{Z}^{n}$, we say that $\alpha$ is a dominant weight if $\lambda_{1} \geq \cdots \geq \lambda_{n}$ holds. By definition, a partition $\alpha \in \mathcal{P}_{n}$ is always dominant. For two partitions $\alpha \in \mathcal{P}_{s}$ and $\beta \in \mathcal{P}_{t}$ with $s+t \leq n$, we put

$$
\alpha \odot \beta=\left(\alpha, 0, \ldots, 0, \beta^{*}\right)=\left(\alpha_{1}, \ldots, \alpha_{s}, 0, \ldots, 0,-\beta_{t}, \ldots,-\beta_{1}\right) \in \mathbb{Z}^{n},
$$


which is also a dominant weight. For a dominant $\lambda$, we denote by $\rho_{\lambda}^{(n)}$ the irreducible finite dimensional representation of $\mathrm{GL}_{n}(\mathbb{C})$ with the highest weight $\lambda$. We often drop the superscript $(n)$, if it is clear from the situation. The contragredient representation of $\rho_{\lambda}$ is denoted by $\rho_{\lambda}^{*}$, and the highest weight of $\rho_{\lambda}^{*}$ by $\lambda^{*}$.

\section{Contraction map AND its Null FiBer}

Let $U_{i}=\mathbb{C}^{m_{i}}(i=1,2)$ and $V=\mathbb{C}^{n}$ be finite dimensional vector spaces, and put

$$
W=V^{*} \otimes U_{1} \oplus\left(U_{2}\right)^{*} \otimes V=\operatorname{Hom}\left(V, U_{1}\right) \times \operatorname{Hom}\left(U_{2}, V\right) .
$$

We consider $W$ as a representation space of $\mathrm{GL}\left(U_{1}\right) \times \mathrm{GL}\left(U_{2}\right) \times \mathrm{GL}(V)$ in a natural manner. Put $H=\mathrm{GL}\left(U_{1}\right) \times \mathrm{GL}\left(U_{2}\right)$ and $K=\mathrm{GL}(V)$ for short. The action of $\left(\left(h_{1}, h_{2}\right), k\right) \in H \times K$ on $\left(f_{1}, f_{2}\right) \in \operatorname{Hom}\left(V, U_{1}\right) \times \operatorname{Hom}\left(U_{2}, V\right)$ is explicitly given by

$$
\left(\left(h_{1}, h_{2}\right), k\right) \cdot\left(f_{1}, f_{2}\right)=\left(h_{1} \circ f_{1} \circ k^{-1}, k \circ f_{2} \circ h_{2}^{-1}\right)
$$

The composite map

$$
\psi: W \rightarrow \operatorname{Hom}\left(U_{2}, U_{1}\right), \quad \psi\left(f_{1}, f_{2}\right)=f_{1} \circ f_{2}
$$

is often called a contraction map. It is an affine quotient map by the action of $K$ onto its image. To state it more precisely, let $r=\min \left\{m_{1}, m_{2},, n\right\}$. Then the image of $\psi$ is

$$
\operatorname{Det}_{r}\left(U_{2}, U_{1}\right)=\left\{f \in \operatorname{Hom}\left(U_{2}, U_{1}\right) \mid \operatorname{rank} f \leq r\right\},
$$

the determinantal variety of rank $r$, so that $W / / K \simeq \operatorname{Det}_{r}\left(U_{2}, U_{1}\right)$.

In general, suppose that a reductive algebraic group $K$ acts on an affine variety (or affine scheme) $X=\operatorname{Spec} A$, where $A$ is a commutative ring. Then an affine quotient (or the categorical quotient in the category of affine schemes) is defined by $X / / K=\operatorname{Spec} A^{K}$, where $A^{K}$ denotes the ring of $K$-invariants. Thus, in our case, an affine quotient is defined to be $W / / K=\operatorname{Spec} \mathbb{C}[W]^{K}$ which is isomorphic to $\operatorname{Det}_{r}\left(U_{2}, U_{1}\right)$ by the classical invariant theory. We abbreviate $\operatorname{Det}_{r}\left(U_{2}, U_{1}\right)$ to $\operatorname{Det}_{r}$, if there is no confusion.

The fiber of $0 \in$ Det $_{r}$ is called the null fiber or null cone, and denoted as

$$
\mathfrak{N}_{K}(W)=\left\{\left(f_{1}, f_{2}\right) \in W \mid f_{1} \circ f_{2}=0\right\}=\left\{\left(f_{1}, f_{2}\right) \in W \mid \operatorname{Im} f_{2} \subset \operatorname{Ker} f_{1}\right\} .
$$

It is easy to identify the $H \times K$-orbit structure of $\mathfrak{N}_{K}(W)$.

Lemma 1.1. Put $\mathfrak{N}_{s, t}^{\circ}=\left\{\left(f_{1}, f_{2}\right) \in \mathfrak{N}_{K}(W) \mid \operatorname{rank} f_{1}=s\right.$, rank $\left.f_{2}=t\right\}$ and $\mathfrak{N}_{s, t}=\overline{\mathfrak{N}_{s, t}^{\circ}}$ its closure. Then

$$
\mathfrak{N}_{K}(W)=\bigsqcup_{0 \leq s \leq m_{1}, 0 \leq t \leq m_{2}} \mathfrak{N}_{s+t \leq n}^{\circ}
$$

gives the $H \times K$-orbit decomposition of the null fiber. We have

$$
\operatorname{dim} \mathfrak{N}_{s, t}=\{n-(s+t)\}(s+t)+s t+m_{1} s+m_{2} t,
$$

and the closure relation is described as follows; $\mathfrak{N}_{s^{\prime}, t^{\prime}} \subset \mathfrak{N}_{s, t}$ if and only if $s^{\prime} \leq s$ and $t^{\prime} \leq t$. Thus, the orbit $\mathfrak{N}_{p, q}^{\circ}$ is open in the null fiber if and only if $p+q=\min \left\{m_{1}+m_{2}, n\right\}$. 
Let $\mathcal{H}_{K}$ denote the space of harmonic polynomials on $W$, which are killed by the positive degree homogeneous $K$-invariant differential operators with constant coefficients (see, e.g., How95] or [GW98] for precise definition).

Lemma 1.2. The space of harmonics decomposes as an $H \times K$-module in the following way. (For the notation, we refer to those in the end of Introduction.)

$$
\mathcal{H}_{K} \simeq \bigoplus_{\substack{\alpha \in \mathcal{P}_{m_{1}}, \beta \in \mathcal{P}_{m_{2}} \\ \ell(\alpha)+\ell(\beta) \leq n}}\left(\rho_{\alpha}^{\left(m_{1}\right) *} \otimes \rho_{\beta}^{\left(m_{2}\right)}\right) \otimes \rho_{\alpha \odot \beta}^{(n)}
$$

Let $J_{K}$ be an ideal of the polynomial ring $\mathbb{C}[W]$ generated by the positive degree homogeneous invariants $\mathbb{C}[W]_{+}^{K} . J_{K}$ is called an invariant ideal. Then it is well known that

$$
\mathbb{C}[W]=\mathcal{H}_{K} \oplus J_{K} \quad(\text { as an } H \times K \text {-module }) .
$$

The following theorem is essentially due to [KV78] and [How89].

Theorem 1.3. The null fiber $\mathfrak{N}_{K}(W)$ is reduced, i.e., the invariant ideal $J_{K}$ is reduced. Thus we have $\mathcal{H}_{K} \simeq \mathbb{C}\left[\mathfrak{N}_{K}(W)\right]$ as an $H \times K$-module. Moreover, we have

(1) If $n \geq m_{1}+m_{2}$, the null fiber $\mathfrak{N}_{K}(W)$ is irreducible, and it coincides with the closure of an open dense $H \times K$-orbit $\mathfrak{N}_{m_{1}, m_{2}}^{\circ}$.

(2) If $1 \leq n<m_{1}+m_{2}$, the null fiber is reducible, and its irreducible decomposition is given by

$$
\mathfrak{N}_{K}(W)=\bigcup_{0 \leq p \leq m_{1}, 0 \leq q \leq m_{2}} \mathfrak{N}_{p, q=n} .
$$

We have an irreducible decomposition of the $H \times K$-module

$$
\left.\mathbb{C}\left[\mathfrak{N}_{p, q}\right] \simeq \bigoplus_{\alpha \in \mathcal{P}_{p}, \beta \in \mathcal{P}_{q}}\left(\rho_{\alpha}^{\left(m_{1}\right) *} \otimes \rho_{\beta}^{\left(m_{2}\right)}\right) \otimes \rho_{\alpha \odot \beta}^{(n)} \quad \text { (as an } H \times K \text {-module }\right) .
$$

For the proof, we refer to Bry93, Theorem3.3].

\section{Resolution of NUll Fibers}

Let $\mathfrak{N}_{p, q}^{\circ}$ be an open orbit in the null fiber. Then it is equivariantly isomorphic to a fiber bundle over certain subvariety of a partial flag variety. We describe the resolution explicitly in the following.

2.1. Stable range case $\left(n \geq m_{1}+m_{2}\right)$. First let us assume $n \geq m_{1}+m_{2}$. In this case, the null fiber is irreducible and $\mathfrak{N}_{m_{1}, m_{2}}^{\circ}$ is the unique open $H \times K$-orbit in $\mathfrak{N}_{K}(W)$. This open orbit has an explicit description,

$$
\begin{aligned}
& \mathfrak{N}_{m_{1}, m_{2}}^{\circ}=\left\{\left(f_{1}, f_{2}\right) \in \operatorname{Hom}\left(V, U_{1}\right) \times \operatorname{Hom}\left(U_{2}, V\right) \mid\right. \\
&\left.\operatorname{Im} f_{2} \subset \operatorname{Ker} f_{1}, \operatorname{rank} f_{1}=m_{1}, \operatorname{rank} f_{2}=m_{2}\right\},
\end{aligned}
$$

so that we can define a surjective map

$$
\zeta_{m_{1}, m_{2}}: \mathfrak{N}_{m_{1}, m_{2}}^{\circ} \ni\left(f_{1}, f_{2}\right) \mapsto\left(\operatorname{Im} f_{2}, \operatorname{Ker} f_{1}\right) \in \mathfrak{F}_{\left(m_{2}, n-m_{1}\right)}(V),
$$


where $\mathfrak{F}_{(s, t)}(V)$ is a partial flag variety consisting of the pair of two subspaces $\left(V_{2}, V_{1}\right)$ of $V$ which satisfies $V_{2} \subset V_{1}$ and $\operatorname{dim} V_{2}=s, \operatorname{dim} V_{1}=t$. We often abbreviate $\mathfrak{F}_{\left(m_{2}, n-m_{1}\right)}(V)$ to $\mathfrak{F}_{m_{2}, m_{1}^{\prime}}$, where $m_{1}^{\prime}=n-m_{1}$. The fiber $\zeta_{m_{1}, m_{2}}^{-1}(x)$ at $x=\left(V_{2}, V_{1}\right) \in \mathfrak{F}_{m_{2}, m_{1}^{\prime}}$ is given by

$$
\zeta_{m_{1}, m_{2}}^{-1}(x)=\left\{\left(f_{1}, f_{2}\right) \mid f_{1}: V / V_{1} \stackrel{\sim}{\rightarrow} U_{1} \text { and } f_{2}: U_{2} \stackrel{\sim}{\rightarrow} V_{2}\right\}
$$

where $f_{1}$ and $f_{2}$ are both isomorphisms, and $f_{1}$ is identified with the map $V \rightarrow V / V_{1} \stackrel{f_{1}}{\rightarrow}$ $U_{1}$, and similarly $f_{2}$ is identified with $U_{2} \stackrel{f_{2}}{\rightarrow} V_{2} \hookrightarrow V$.

Let us consider the tautological bundle $\mathcal{T}_{m_{2}}$ on $\mathfrak{F}_{m_{2}, m_{1}^{\prime}}$. It is a vector subbundle of the trivial bundle $\mathfrak{F}_{m_{2}, m_{1}^{\prime}} \times V$ with the fiber $V_{2}$ at $x=\left(V_{2}, V_{1}\right) \in \mathfrak{F}_{m_{2}, m_{1}^{\prime}}$. Similarly, we can define $\mathcal{T}_{m_{1}^{\prime}}$ for which the fiber at $x$ is $V_{1}$, and define the quotient bundle by

$$
\mathcal{Q}_{m_{1}^{\prime}}=\left(\mathfrak{F}_{m_{2}, m_{1}^{\prime}} \times V\right) / \mathcal{T}_{m_{1}^{\prime}}
$$

Also we denote by $\mathcal{U}_{i}$ the trivial bundle $\mathfrak{F}_{m_{2}, m_{1}^{\prime}} \times U_{i}(i=1,2)$. From the description of the fiber $\zeta_{m_{1}, m_{2}}^{-1}(x)$ in (2.1), $\mathfrak{N}_{m_{1}, m_{2}}^{\circ}$ can be identified with the subbundle of the vector bundle $\operatorname{Hom}\left(\mathcal{Q}_{m_{1}^{\prime}}, \mathcal{U}_{1}\right) \times \operatorname{Hom}\left(\mathcal{U}_{2}, \mathcal{T}_{m_{2}}\right)$. We denote this subbundle by $\operatorname{Iso}\left(\mathcal{Q}_{m_{1}^{\prime}}, \mathcal{U}_{1}\right) \times \operatorname{Iso}\left(\mathcal{U}_{2}, \mathcal{T}_{m_{2}}\right)$. Namely it is explicitly given as

$$
\begin{aligned}
& \operatorname{Iso}\left(\mathcal{Q}_{m_{1}^{\prime}}, \mathcal{U}_{1}\right) \times \operatorname{Iso}\left(\mathcal{U}_{2}, \mathcal{T}_{m_{2}}\right) \\
& \quad=\left\{\left(x ;\left(f_{1}, f_{2}\right)\right) \in \operatorname{Hom}\left(\mathcal{Q}_{m_{1}^{\prime}}, \mathcal{U}_{1}\right) \times \operatorname{Hom}\left(\mathcal{U}_{2}, \mathcal{T}_{m_{2}}\right) \mid f_{1} \text { and } f_{2} \text { are isomorphisms }\right\}
\end{aligned}
$$

Proposition 2.1. We assume the stable range condition $n \geq m_{1}+m_{2}$, and put $m_{1}^{\prime}=$ $n-m_{1}$.

(1) There is an $H \times K$-equivariant isomorphism

$$
\mathfrak{N}_{m_{1}, m_{2}}^{\circ} \simeq \operatorname{Iso}\left(\mathcal{Q}_{m_{1}^{\prime}}, \mathcal{U}_{1}\right) \times \operatorname{Iso}\left(\mathcal{U}_{2}, \mathcal{T}_{m_{2}}\right)
$$

(2) The map

$$
\nu_{m_{1}, m_{2}}: \operatorname{Hom}\left(\mathcal{Q}_{m_{1}^{\prime}}, \mathcal{U}_{1}\right) \times \operatorname{Hom}\left(\mathcal{U}_{2}, \mathcal{T}_{m_{2}}\right) \rightarrow \mathfrak{N}_{K}(W), \quad \nu_{m_{1}, m_{2}}\left(\left(x ;\left(f_{1}, f_{2}\right)\right)\right)=\left(f_{1}, f_{2}\right)
$$

gives an equivariant resolution of singularities of the null fiber.

Proof. The first statement is obvious from the construction. Let us consider the second statement. We say the map $\nu: X \rightarrow Y$ is a resolution of singularities of $Y$ if (a) $X$ is smooth, (b) $\nu$ is a proper birational map, and (c) it is one to one precisely on the smooth points of $Y$. In our case, (a) and the birationality in (b) are clear, and that the map $\nu_{m_{1}, m_{2}}$ is proper follows because $\operatorname{Hom}\left(\mathcal{Q}_{m_{1}^{\prime}}, \mathcal{U}_{1}\right) \times \operatorname{Hom}\left(\mathcal{U}_{2}, \mathcal{T}_{m_{2}}\right)$ is a vector bundle over a projective variety (see, e.g., [Har77, Corollary 4.8 and Theorem 4.9]). The condition (c) is also easy to verify. Note that $\mathfrak{N}_{m_{1}, m_{2}}^{\circ}$ is precisely the set of smooth points of the null fiber, which can be checked by the explicit calculations of the rank of differentials of defining equations. 
2.2. Outside of the stable range $\left(n<m_{1}+m_{2}\right)$. In this subsection, we assume that $n<m_{1}+m_{2}$. Take an open $H \times K$-orbit $\mathfrak{N}_{p, q}^{\circ}(p+q=n)$ in the null fiber. In this case, for $\left(f_{1}, f_{2}\right) \in \mathfrak{N}_{p, q}^{\circ}$, the image of $f_{2}$ and the kernel of $f_{1}$ coincide with each other since $p+q=n$, and it gives a $q$-dimensional subspace $V_{2}$ of $V$. Thus, if we denote the Grassmann variety of $q$-dimensional subspaces in $V$ by $\operatorname{Grass}_{q}(V)$, there is a $K$-equivariant map

$$
\zeta_{p, q}: \mathfrak{N}_{p, q}^{\circ} \rightarrow \operatorname{Grass}_{q}(V)
$$

which sends $\left(f_{1}, f_{2}\right) \in \mathfrak{N}_{p, q}^{\circ}$ to $\operatorname{Im} f_{2}=\operatorname{Ker} f_{1} \in \operatorname{Grass}_{q}(V)$. This map is surjective, and $\mathfrak{N}_{p, q}^{\circ}$ is turned out to be a fiber bundle over $\operatorname{Grass}_{q}(V)$ with the fiber at $x=V_{2} \in \operatorname{Grass}_{q}(V)$

$$
\zeta_{p, q}^{-1}(x)=\left\{\left(f_{1}, f_{2}\right) \mid f_{1}: V / V_{2} \hookrightarrow U_{1} \text { and } f_{2}: U_{2} \rightarrow V_{2}\right\},
$$

where $f_{1}$ is injective and $f_{2}$ is surjective.

We denote the tautological bundle on $\operatorname{Grass}_{q}(V)$ by $\mathcal{T}_{q}$, and the quotient bundle $\left(\operatorname{Grass}_{q}(V) \times V\right) / \mathcal{T}_{q}$ by $\mathcal{Q}_{q}$. As in the former subsection, $\mathcal{U}_{i}=\operatorname{Grass}_{q}(V) \times U_{i}(i=1,2)$ denotes the trivial bundle with the fiber $U_{i}$. We denote by $\operatorname{Hom}^{\circ}\left(\mathcal{Q}_{q}, \mathcal{U}_{1}\right)$ and $\operatorname{Hom}^{\circ}\left(\mathcal{U}_{2}, \mathcal{T}_{q}\right)$ the subbundle of the full rank maps in $\operatorname{Hom}\left(\mathcal{Q}_{q}, \mathcal{U}_{1}\right)$ and $\operatorname{Hom}\left(\mathcal{U}_{2}, \mathcal{T}_{q}\right)$ respectively.

Proposition 2.2. Let us assume that $n<m_{1}+m_{2}$.

(1) An open orbit $\mathfrak{N}_{p, q}^{\circ}\left(p+q=n, 0 \leq p \leq m_{1}, 0 \leq q \leq m_{2}\right)$ in $\mathfrak{N}_{K}(W)$ is $H \times K$ equivariantly isomorphic to $\operatorname{Hom}^{\circ}\left(\mathcal{Q}_{q}, \mathcal{U}_{1}\right) \times \operatorname{Hom}^{\circ}\left(\mathcal{U}_{2}, \mathcal{T}_{q}\right)$.

(2) The map

$$
\nu_{p, q}: \operatorname{Hom}\left(\mathcal{Q}_{q}, \mathcal{U}_{1}\right) \times \operatorname{Hom}\left(\mathcal{U}_{2}, \mathcal{T}_{q}\right) \rightarrow \mathfrak{N}_{p, q}, \quad \nu_{p, q}\left(x ;\left(f_{1}, f_{2}\right)\right)=\left(f_{1}, f_{2}\right)
$$

gives an $H \times K$-equivariant resolution of singularities of the irreducible component $\mathfrak{N}_{p, q}$ of the null fiber.

The proof of this proposition is completely similar to that of Proposition 2.1. So we omit it.

We will denote the resolution of singularities in Propositions 2.1 and 2.2 by

$$
\nu_{p, q}: \widetilde{\mathfrak{N}}_{p, q} \rightarrow \mathfrak{N}_{p, q}, \quad \widetilde{\mathfrak{N}}_{p, q}=\operatorname{Hom}\left(\mathcal{Q}_{p^{\prime}}, \mathcal{U}_{1}\right) \times \operatorname{Hom}\left(\mathcal{U}_{2}, \mathcal{T}_{q}\right)
$$

in the following $\left(p^{\prime}=n-p\right)$.

2.3. Reduction to the stable range lift. Let us assume that we are still in the outside of the stable range, i.e., assume $n<m_{1}+m_{2}$. The resolution of $\mathfrak{N}_{p, q}(p+q=n)$ above can be reduced to the stable range case. Let us explain it.

For $0 \leq p \leq m_{1}, 0 \leq q \leq m_{2}$ which satisfies $n=p+q$, we take a subspace $U^{p} \subset$ $U_{1}, U^{q} \subset U_{2}$ such that $\operatorname{dim} U^{p}=p$ and $\operatorname{dim} U^{q}=q$. Then one can play the same game for $W^{p, q}=\operatorname{Hom}\left(V, U^{p}\right) \times \operatorname{Hom}\left(U^{q}, V\right)$ instead of $W$, with $H=\operatorname{GL}\left(U_{1}\right) \times \operatorname{GL}\left(U_{2}\right)$ replaced by $\operatorname{GL}\left(U^{p}\right) \times \operatorname{GL}\left(U^{q}\right)$. We can consider naturally $W^{p, q}$ as a subspace of $W$, and the intersection $\mathfrak{N}_{p, q} \cap W^{p, q}$ is exactly the null fiber of the quotient map $W^{p, q} \rightarrow W^{p, q} / / K=$ $\operatorname{Hom}\left(U^{q}, U^{p}\right)$ in the stable range. 
Now we return to the $H \times K$-orbit $\mathfrak{N}_{p, q}^{\circ}$ in $W$. To be sure, we again take $H$ as $\mathrm{GL}\left(U_{1}\right) \times$ $\operatorname{GL}\left(U_{2}\right)$. For $\left(f_{1}, f_{2}\right) \in \mathfrak{N}_{p, q}^{\circ}$, we can associate $\left(\operatorname{Im} f_{1}, \operatorname{Ker} f_{2}\right) \in \operatorname{Grass}_{p}\left(U_{1}\right) \times \operatorname{Grass}_{p}\left(U_{2}\right)$. Notice that $\left(\operatorname{Ker} f_{2}\right)^{\perp}=\operatorname{Im} f_{2}^{*}$, which is of dimension $q=n-p$. The projection $\mathfrak{N}_{p, q}^{\circ} \rightarrow$ $\operatorname{Grass}_{p}\left(U_{1}\right) \times \operatorname{Grass}_{p}\left(U_{2}\right)$ given above is surjective, and it gives $\mathfrak{N}_{p, q}^{\circ}$ a structure of a fiber bundle over the product of two Grassmannian. In this picture, $\mathfrak{N}_{p, q}^{\circ}$ is a subbundle of the vector bundle

$$
\operatorname{Hom}\left(\mathcal{V}, \mathcal{T}_{p}\left(U_{1}\right)\right) \times \operatorname{Hom}\left(\mathcal{Q}_{p}\left(U_{2}\right), \mathcal{V}\right) \rightarrow \operatorname{Grass}_{p}\left(U_{1}\right) \times \operatorname{Grass}_{p}\left(U_{2}\right)
$$

where $\mathcal{V}$ denotes the trivial bundle with the fiber $V$. Thus $\mathfrak{N}_{p, q}^{\circ}$ can be identified with

$$
\begin{aligned}
\mathfrak{N}_{p, q}^{\circ}=\left\{\left(x ;\left(f_{1}, f_{2}\right)\right) \in \operatorname{Hom}\left(\mathcal{V}, \mathcal{T}_{p}\left(U_{1}\right)\right) \times \operatorname{Hom}\left(\mathcal{Q}_{p}\left(U_{2}\right), \mathcal{V}\right) \mid\right. \\
\left.f_{1} \text { and } f_{2} \text { are of full rank and } f_{1} \circ f_{2}=0\right\}
\end{aligned}
$$

which means that it is a fiber bundle over $\operatorname{Grass}_{p}\left(U_{1}\right) \times \operatorname{Grass}_{p}\left(U_{2}\right)$ with a fiber isomorphic to the dense orbit in the null fiber of the reduced quotient map $W^{p, q} \rightarrow \operatorname{Hom}\left(U^{q}, U^{p}\right)$. We already know that the null fiber of the map $W^{p, q} \rightarrow \operatorname{Hom}\left(U^{q}, U^{p}\right)$ has a resolution of singularities

$$
\operatorname{Hom}\left(\mathcal{Q}_{p^{\prime}}(V), U^{p}\right) \times \operatorname{Hom}\left(U^{q}, \mathcal{T}_{q}(V)\right) \rightarrow \operatorname{Grass}_{q}(V) \quad\left(p^{\prime}=n-p=q\right)
$$

(see Equation (2.3) ). Thus we have a simultaneous resolution of $\mathfrak{N}_{p, q}$ by the vector bundle

$$
\begin{aligned}
\operatorname{Hom}\left(\mathcal{Q}_{p^{\prime}}(V), \mathcal{T}_{p}\left(U_{1}\right)\right) \times \operatorname{Hom}\left(\mathcal{Q}_{q^{\prime}}\left(U_{2}\right), \mathcal{T}_{q}(V)\right) & \\
& \rightarrow \operatorname{Grass}_{q}(V) \times \operatorname{Grass}_{p}\left(U_{1}\right) \times \operatorname{Grass}_{p}\left(U_{2}\right) .
\end{aligned}
$$

The explicit resolution map is given, for $x=\left(V_{0}, V_{1}, V_{2}\right) \in \operatorname{Grass}_{q}(V) \times \operatorname{Grass}_{p}\left(U_{1}\right) \times$ $\operatorname{Grass}_{p}\left(U_{2}\right)$, by

$$
\left(x ;\left(F_{1}, F_{2}\right)\right) \mapsto\left(f_{1}, f_{2}\right) \quad \text { where } \quad\left\{\begin{array}{l}
f_{1}: V \rightarrow V / V_{0} \stackrel{F_{1}}{\rightarrow} V_{1} \hookrightarrow U_{1} \\
f_{2}: U_{2} \rightarrow U_{2} / V_{2} \stackrel{F_{2}}{\rightarrow} V_{0} \hookrightarrow V
\end{array}\right.
$$

We denote this simultaneous resolution by

$$
\hat{\nu}_{p, q}: \widehat{\mathfrak{N}}_{p, q}=\operatorname{Hom}\left(\mathcal{Q}_{p^{\prime}}(V), \mathcal{T}_{p}\left(U_{1}\right)\right) \times \operatorname{Hom}\left(\mathcal{Q}_{q^{\prime}}\left(U_{2}\right), \mathcal{T}_{q}(V)\right) \rightarrow \mathfrak{N}_{p, q}
$$

Thus we have established the following theorem, which shows that indeed $\widehat{\mathfrak{N}}_{p, q}$ is the collection of the resolutions of the null fibers in the stable range, which is parametrized by $\operatorname{Grass}_{p}\left(U_{1}\right) \times \operatorname{Grass}_{p}\left(U_{2}\right)$. 
Theorem 2.3. (1) There is a natural surjective, birational map $f_{p, q}: \widehat{\mathfrak{N}}_{p, q} \rightarrow \widetilde{\mathfrak{N}}_{p, q}$ such that $\hat{\nu}_{p, q}$ factors to $\hat{\nu}_{p, q}=\nu_{p, q} \circ f_{p, q}$. Moreover, the following diagram commutes.

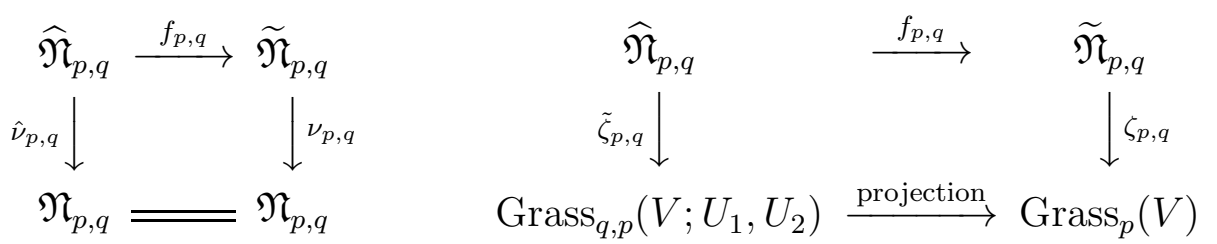

where $\operatorname{Grass}_{q, p}\left(V ; U_{1}, U_{2}\right)=\operatorname{Grass}_{q}(V) \times \operatorname{Grass}_{p}\left(U_{1}\right) \times \operatorname{Grass}_{p}\left(U_{2}\right)$.

(2) Fix a point $x=\left(X_{1}, X_{2}\right) \in \operatorname{Grass}_{p}\left(U_{1}\right) \times \operatorname{Grass}_{p}\left(U_{2}\right)$ and put $U^{p}=X_{1}, U^{q}=U_{2} / X_{2}$. Then

$$
\mathfrak{N}_{p, q} \cap W^{p, q}=\left\{\left(f_{1}, f_{2}\right) \in \mathfrak{N}_{K}(W) \mid \operatorname{Im} f_{1} \subset X_{1}, X_{2} \subset \text { Ker } f_{2}\right\}
$$

is isomorphic to the null fiber of the contraction map $W^{p, q} \rightarrow \operatorname{Hom}\left(U^{p}, U^{q}\right)$, and the map

$$
\tilde{\zeta}_{p, q}^{-1}\left(\operatorname{Grass}_{q}(V) \times\{x\}\right) \rightarrow \mathfrak{N}_{p, q} \cap W^{p, q}
$$

gives a resolution of singularities of the null cone $\mathfrak{N}_{p, q} \cap W^{p, q}$. Moreover, $f_{p, q}$ restricted to $\tilde{\zeta}_{p, q}^{-1}\left(\operatorname{Grass}_{p}(V) \times\{x\}\right)$ is an isomorphism into $\widetilde{\mathfrak{N}}_{p, q}$.

\section{DUAL PAIR AND MOMENT MAPS}

In this section, we briefly recall the properties of moment maps and the theta lifting of nilpotent orbits.

Let us consider the dual pair $\left(G_{\mathbb{R}}, G_{\mathbb{R}}^{\prime}\right)=\left(\operatorname{Sp}_{2 n}(\mathbb{R}), \mathrm{O}_{m_{1}, m_{2}}(\mathbb{R})\right)$ in $\mathbb{G}_{\mathbb{R}}=\operatorname{Sp}_{2 N}(\mathbb{R})$ where $N=n\left(m_{1}+m_{2}\right)$ (see [How85] for the definition of the dual pair). Here $G_{\mathbb{R}}=\mathrm{Sp}_{2 n}(\mathbb{R})$ is realized as the group of linear transformations on $\mathbb{R}^{2 n}$ which preserve a given symplectic form. Also $G_{\mathbb{R}}^{\prime}=\mathrm{O}_{m_{1}, m_{2}}(\mathbb{R})$ is the indefinite orthogonal group realized on a quadratic space $\mathbb{R}^{m_{1}, m_{2}}$ with the signature $\left(m_{1}, m_{2}\right)$. Then the tensor product $W=\mathbb{R}^{2 n} \otimes \mathbb{R}^{m_{1}, m_{2}}$ has a natural symplectic form which is the product of the symplectic form on $\mathbb{R}^{2 n}$ and the symmetric form on $\mathbb{R}^{m_{1}, m_{2}}$. We denote this symplectic form by $\langle$,$\rangle . Thus \mathbb{G}_{\mathbb{R}}=\operatorname{Sp}_{2 N}(\mathbb{R})$ defined by $\langle$,$\rangle contains \operatorname{Sp}_{2 n}(\mathbb{R})$ and $\mathrm{O}_{m_{1}, m_{2}}(\mathbb{R})$ as subgroups and they are mutually commutant to each other, i.e., they form a dual pair.

Let us introduce a complex structure on the symplectic space $W$.

Take a complete polarization $W=X \oplus Y$. Since $X$ and $Y$ are totally isotropic and the symplectic form is non-degenerate, there is a complete pairing on $X \times Y$. Thus we can identify $Y=X^{*}$. Let us fix a standard Euclidean inner product on $X$. Then it also gives an identification $X \simeq X^{*}$, and we get an isomorphism $X \rightarrow X^{*} \rightarrow Y$. We denote this isomorphism by $\eta$.

Let us denote the complexification of $W$ by $W_{\mathbb{C}}=W \otimes_{\mathbb{R}} \mathbb{C}$ (we use the same notation for the complexification for any real vector space). Then $\eta: X \rightarrow Y$ extends to $\eta: X_{\mathbb{C}} \rightarrow Y_{\mathbb{C}}$ linearly. Put

$$
L^{+}=\left\{x+i \eta(x) \mid x \in X_{\mathbb{C}}\right\} .
$$


It is easy to check that $L^{+}$is Lagrangian in $W_{\mathbb{C}}$ with respect to the complexified symplectic form. Then we get an isomorphism $\gamma: L^{+} \rightarrow W$ over $\mathbb{R}$ defined by $\gamma(z)=\operatorname{Re} z$, where Re $z$ denotes the real part of $z$. By this isomorphism, we can identify $X_{\mathbb{C}} \simeq L^{+} \simeq W$, and $W$ enjoys a complex structure coming from this identification. Also we define a Hermitian inner product on $X_{\mathbb{C}}$ using the Euclidean inner product on $X$, and transfer it to $W$. Denote this inner product by $($,$) . Then the symplectic form \langle$,$\rangle on W$ is recovered from it as $\langle v, w\rangle=-\operatorname{Im}(v, w)$. Thus, the unitary group $\mathrm{U}(W)$ with respect to $\left(\right.$, ) preserves the symplectic form, hence it is a maximal compact subgroup $\mathbb{K}_{\mathbb{R}}$ of $\mathbb{G}_{\mathbb{R}}$. Also its complexification $\mathbb{K} \simeq \mathrm{GL}_{N}(\mathbb{C})$ naturally acts on $W, X_{\mathbb{C}}$ and $L^{+}$through the above isomorphism, and this in turn determines an embedding of $\mathbb{K}$ into $\mathbb{G}:=\mathrm{Sp}_{2 N}(\mathbb{C})$.

Since the vector representation of $\operatorname{Sp}_{2 N}(\mathbb{C})$ on $W_{\mathbb{C}}$ is Hamiltonian, there is a moment map $\mu: W_{\mathbb{C}} \rightarrow \mathfrak{s p}_{2 N}(\mathbb{C})^{*}$, the algebraic dual of the Lie algebra of $\operatorname{Sp}_{2 N}(\mathbb{C})$. The map $\mu$ can be described explicitly by the formula $\mu(w)(X)=\frac{1}{2}\langle X w, w\rangle\left(w \in W, X \in \mathfrak{s p}_{2 N}(\mathbb{C})\right)$. Then it is easy to see that the image $\operatorname{Im} \mu$ coincides with the closure of the minimal nilpotent coadjoint orbit $\overline{\mathbb{O}_{\min } \mathbb{C}}$. The moment map restricted to $L^{+}$has an image which is orthogonal to $\operatorname{Lie}\left(\mathbb{K}_{\mathbb{R}}\right)_{\mathbb{C}}=\mathfrak{K}$. If we denote the complexified Cartan decomposition with respect to $\mathbb{K}_{\mathbb{R}}$ by $\mathfrak{G}=\mathfrak{K} \oplus \mathfrak{P}$, then

$$
\mu\left(L^{+}\right)=\overline{\mathbb{O}_{\min }^{+}} \subset \overline{\mathbb{O}_{\min \mathbb{C}}} \cap \mathfrak{P}^{*},
$$

where $\mathbb{O}_{\text {min }}^{+}$is one of the minimal nilpotent $\mathbb{K}$-orbits in $\mathfrak{P}^{*}$. Note that $\mu$ is $\mathbb{K}$-equivariant.

Now take a complete polarization $\mathbb{R}^{2 n}=X_{0} \oplus Y_{0}$. Then $X=X_{0} \otimes \mathbb{R}^{m_{1}, m_{2}}$ is a Lagrangian subspace in $W$ and, if we define $Y$ similarly, $W=X \oplus Y$ gives a complete polarization. Using this $X$, we follow the above recipe to construct a moment map. We take maximal compact subgroups $K_{\mathbb{R}}, K_{\mathbb{R}}^{\prime}$ of $G_{\mathbb{R}}, G_{\mathbb{R}}^{\prime}$ respectively inside $\mathbb{K}_{\mathbb{R}}$, hence we get a compatible (complexified) Cartan decomposition

$$
\mathfrak{g}=\mathfrak{k} \oplus \mathfrak{p}, \quad \mathfrak{k} \subset \mathfrak{K}, \quad \mathfrak{p} \subset \mathfrak{P} .
$$

Similarly we define $\mathfrak{g}^{\prime}=\mathfrak{k}^{\prime} \oplus \mathfrak{p}^{\prime}$ to be a Cartan decomposition for $G_{\mathbb{R}}^{\prime}$. Then we can define a map

$$
\varphi: W \simeq X_{\mathbb{C}} \stackrel{\sim}{\rightarrow} L^{+} \stackrel{\mu}{\rightarrow} \overline{\mathbb{O}_{\text {min }}^{+}} \stackrel{\text { res. }}{\longrightarrow} \mathfrak{p}^{*}
$$

where the last map is the restriction of $\mathfrak{P}^{*}$ to the subspace $\mathfrak{p}$. This is a $K:=K_{\mathbb{C}^{-}}$ equivariant map. Since $K^{\prime}:=K_{\mathbb{C}}^{\prime}$ acts trivially on $\mathfrak{p}$, the map $\varphi$ is $K^{\prime}$-invariant. Similarly we can define a $K^{\prime}$-equivariant map $\psi: W \rightarrow \mathfrak{p}^{\prime *}$ which is $K$-invariant. Thus we have a double fibration of the complex vector space $W$.

Remark 3.1. Since the action of $G_{\mathbb{R}}$ or $G_{\mathbb{R}}^{\prime}$ on $W$ is Hamiltonian, we can define moment maps $\mu: W_{\mathbb{C}} \rightarrow \mathfrak{g}^{*}$ (or $\mathfrak{g}^{\prime *}$ respectively). If we restrict this moment map to $W \simeq L^{+}$, then they coincide with $\varphi$ or $\psi$ respectively.

If we denote $V=\left(X_{0}\right)_{\mathbb{C}}=\mathbb{C}^{n}$ and $U_{i}=\mathbb{C}^{m_{i}}(i=1,2)$ as in \$1, we can identify $W=\operatorname{Hom}\left(V, U_{1}\right) \oplus \operatorname{Hom}\left(U_{2}, V\right)$ and the map $\psi$ coincides with the contraction map (see (1.1)). 
Let us describe the map $\varphi: W \rightarrow \mathfrak{p}^{*}$. We shall identify $\mathfrak{p}^{*}$ and $\mathfrak{p}$ by the Killing form, and note that there is a $K$-stable decomposition $\mathfrak{p} \simeq \mathfrak{p}_{+} \oplus \mathfrak{p}_{-}$, where $\mathfrak{p}_{+} \simeq \operatorname{Sym}_{n}(\mathbb{C})=\operatorname{Sym}(V)$ and $\mathfrak{p}_{-} \simeq \operatorname{Sym}\left(V^{*}\right)$. Here we denote by $\operatorname{Sym}_{n}(\mathbb{C})$ the space of $n \times n$-symmetric matrices, and $\operatorname{Sym}(V)$ denotes the space of symmetric tensors of degree two. Take $\left(f_{1}, f_{2}\right) \in$ $\operatorname{Hom}\left(V, U_{1}\right) \oplus \operatorname{Hom}\left(U_{2}, V\right)=W$. We identify $U_{i}$ with $U_{i}^{*}$ by the symmetric form on $U_{i}(i=1,2)$, which comes from the complexification of the quadratic form on $\mathbb{R}^{m_{1}, m_{2}}$. Thus we have $f_{1}^{*} \circ f_{1}$ by composing

$$
V \stackrel{f_{1}}{\rightarrow} U_{1} \simeq U_{1}^{*} \stackrel{f_{1}^{*}}{\rightarrow} V^{*}
$$

It is clear that $f_{1}^{*} \circ f_{1} \in \operatorname{Sym}\left(V^{*}\right)$. Similarly we get $f_{2} \circ f_{2}^{*} \in \operatorname{Sym}(V)$. Now $\varphi$ is given by

$$
\varphi\left(f_{1}, f_{2}\right)=\left(f_{1}^{*} \circ f_{1}, f_{2} \circ f_{2}^{*}\right) \in \operatorname{Sym}\left(V^{*}\right) \oplus \operatorname{Sym}(V) .
$$

The map $\varphi$ is an affine quotient map from $W$ onto its image by the action of $K^{\prime}=$ $\mathrm{O}_{m_{1}}(\mathbb{C}) \times \mathrm{O}_{m_{2}}(\mathbb{C})$. The image is the determinantal variety in the symmetric matrices (or tensors).

Theorem 3.2. Assume the stable range condition $n \geq m_{1}+m_{2}$. For any $K^{\prime}$-orbit $\mathbb{O}^{\prime}$ in $\mathfrak{p}^{\prime}$, there exists a single $K$-orbit $\mathbb{O}$ in $\mathfrak{p}$ such that $\varphi\left(\psi^{-1}\left(\overline{\mathbb{O}^{\prime}}\right)=\overline{\mathbb{O}}\right.$ holds. This correspondence, called $\theta$-lifting, establishes an injection from the set of $K^{\prime}$-orbits into that of $K$-orbits: $\theta: \mathfrak{p}^{\prime} / K^{\prime} \hookrightarrow \mathfrak{p} / K$. Moreover, we have

(1) The $\theta$-lifting carries a nilpotent $K^{\prime}$-orbit to a nilpotent $K$-orbit.

(2) The affine quotient $\psi^{-1}\left(\overline{\mathbb{O}^{\prime}}\right) / / K^{\prime}$ is isomorphic to $\overline{\mathbb{O}}$.

This theorem (in wider context) is obtained independently by [NOZ06] and [DKP02, DKP05] for nilpotent orbits. Also it is noticed by Takuya Ohta (private communication) and the author ([Nis05]) that the lifting map can be defined for arbitrary orbits.

For $p+q \leq n$, we denote by $\mathbb{O}_{p, q}$ the $\theta$-lifting of the trivial orbit $\{0\} \subset \mathfrak{p}^{\prime}$. This is a two step nilpotent $K$-orbit of the shape $2^{p+q} \cdot 1^{2(n-(p+q))}$. The signed Young diagram is given as $(+-)^{p}(-+)^{q}(+)^{n-(p+q)}(-)^{n-(p+q)}$. (See [Nis00] for the more detailed properties of this orbit.)

\section{INTERSECTION OF LAGRANGIAN FLAG VARIETIES}

From now on, we assume $n=p=q$, i.e., $\operatorname{dim} V$ is equal to $\operatorname{dim} U_{1}=\operatorname{dim} U_{2}$. This case is called equal rank case. Henceforth, we will denote $U=U_{1}=U_{2}$.

First we introduce two nondegenerate bilinear forms on $\mathbb{V}:=V \oplus V^{*}$, where $V^{*}=$ $\operatorname{Hom}(V, \mathbb{C})$ is the algebraic dual. One $\left\langle\langle,\rangle_{+}\right.$of the two bilinear forms is a symmetric form, and the other $\left\langle\langle,\rangle_{-}\right.$is a symplectic (or alternating) one. We put

$$
\langle\langle\xi, v\rangle\rangle_{ \pm}=\xi(v) \quad \text { for } \xi \in V^{*} \text { and } v \in V .
$$

Also the subspaces $V$ and $V^{*}$ are assumed to be totally isotropic, so that $\mathbb{V}=V \oplus V^{*}$ is a joint polar decomposition for the both $\left\langle\langle,\rangle_{ \pm}\right.$. Recall that $W=\operatorname{Hom}(V, U) \times \operatorname{Hom}(U, V)$. 
KYO NISHIYAMA

Lemma 4.1. The pair of maps $\left(f_{1}, f_{2}\right) \in W$ belongs to the null fiber $\mathfrak{N}_{K}(W)$ if and only if $\operatorname{Im} f_{2} \oplus \operatorname{Im} f_{1}^{*} \subset \mathbb{V}$ is a joint isotropic subspace with respect to $\left\langle\langle,\rangle_{ \pm}\right.$, where $f_{1}^{*}: U^{*} \rightarrow V^{*}$ is the adjoint map of $f_{1}$.

For $w=\left(f_{1}, f_{2}\right) \in W$, we put $\mathbb{U}_{w}=\operatorname{Im} f_{2} \oplus \operatorname{Im} f_{1}^{*}$, which is a subspace of $\mathbb{V}$. According to the lemma above, $\mathbb{U}_{w}$ is totally isotropic for both $\langle\langle,\rangle\rangle_{ \pm}$if and only if $w \in \mathfrak{N}_{K}(W)$. Moreover, $\mathbb{U}_{w}$ is a joint Lagrangian subspace (i.e., a maximal totally isotropic space for both of $\langle\langle,\rangle\rangle_{ \pm}$) if and only if $w$ belongs to one of the open orbit $\mathfrak{N}_{p, q}^{\circ}$ where $p+q=n$.

Let us denote by $\mathcal{Z}$ a closed subvariety of $\operatorname{Grass}_{n}(\mathbb{V})$ of all the joint Lagrangian subspaces in $\mathbb{V}$.

Lemma 4.2. (1) If $\mathbb{L}$ is a joint Lagrangian subspace of $\mathbb{V}$, it is decomposed into a direct sum $\mathbb{L}=\mathbb{L} \cap V \oplus \mathbb{L} \cap V^{*}$. Thus we have a disjoint decomposition of $\mathcal{Z}$ as

$$
\mathcal{Z}=\bigsqcup_{p+q=n} \mathcal{Z}_{p, q}, \quad \mathcal{Z}_{p, q}=\left\{\mathbb{L} \in \mathcal{Z} \mid \operatorname{dim} \mathbb{L} \cap V=p, \operatorname{dim} \mathbb{L} \cap V^{*}=q\right\}
$$

Each $\mathcal{Z}_{p, q}$ is a single closed $K$-orbit, hence an irreducible smooth projective variety. Moreover, there is a $K$-equivariant isomorphism

$$
\mathcal{Z}_{p, q} \stackrel{\sim}{\longrightarrow} \operatorname{Grass}_{p}(V), \quad \mathbb{L} \mapsto \mathbb{L} \cap V .
$$

(2) The map $z_{p, q}: \mathfrak{N}_{p, q}^{\circ} \rightarrow \mathcal{Z}_{p, q}$ defined by $z_{p, q}(w)=\mathbb{U}_{w}\left(w \in \mathfrak{N}_{p, q}^{\circ}\right)$ is surjective.

Proof. Let $\mathbb{L}$ be a joint Lagrangian subspace. We define an involution $\tau$ on $\mathbb{V}$ by $\left.\tau\right|_{V}=\operatorname{id}_{V}$ and $\left.\tau\right|_{V^{*}}=-\mathrm{id}_{V^{*}}$. If $\mathbb{L}$ is stable under $\tau$, then $\mathbb{L}$ is homogeneous with respect to the direct sum decomposition $\mathbb{V}=V \oplus V^{*}$, and we get the decomposition $\mathbb{L}=\mathbb{L} \cap V \oplus \mathbb{L} \cap V^{*}$. Take $u+\xi, v+\eta \in \mathbb{L}$, where $u, v \in V$ and $\xi, \eta \in V^{*}$. Then we have

$$
\begin{aligned}
\langle\langle u+\xi, \tau(v+\eta)\rangle\rangle_{-} & =\langle\langle u+\xi, v-\eta\rangle\rangle_{-}=-\langle\langle u, \eta\rangle\rangle_{-}+\langle\langle\xi, v\rangle\rangle_{-} \\
& =\eta(u)+\xi(v)=\langle\langle u, \eta\rangle\rangle_{+}+\langle\langle\xi, v\rangle\rangle_{+}=\langle\langle u+\xi, v+\eta\rangle\rangle_{+}=0
\end{aligned}
$$

Thus $\tau(v+\eta)$ is orthogonal to $\mathbb{L}$ with respect to the symplectic form $\langle\langle,\rangle\rangle_{-}$. Since $\mathbb{L}$ is maximally isotropic, this implies $\tau(v+\eta) \in \mathbb{L}$.

Let us write $\mathbb{L}_{E}=\mathbb{L} \cap E$ for a subspace $E$ in $\mathbb{V}$. Then an $n$-dimensional subspace $\mathbb{L}=\mathbb{L}_{V} \oplus \mathbb{L}_{V^{*}}$ is joint Lagrangian if and only if $\mathbb{L}_{V^{*}}=\left(\mathbb{L}_{V}\right)^{\perp}$. Thus $\mathcal{Z}_{p, q}$ is isomorphic to $\operatorname{Grass}_{p}(V)$, which proves it is a single $K$-orbit at the same time. Since $\mathcal{Z}_{p, q}=\mathcal{Z} \cap$ $\operatorname{Grass}_{p}(V) \times \operatorname{Grass}_{q}\left(V^{*}\right)$, it is closed. Now we have proved (11).

The second assertion is clear from Lemma 4.1.

Notice that $\mathfrak{X}:=\operatorname{Grass}_{n}(\mathbb{V}) \simeq \mathrm{GL}(\mathbb{V}) / P_{n}$, where $P_{n}=\operatorname{Stab}_{\mathrm{GL}(\mathbb{V})}(V)$ denotes a maximal parabolic subgroup which stabilizes $V$. Then $\mathfrak{X}^{+}:=\mathrm{O}(\mathbb{V}) \cdot e P_{n} \subset \mathfrak{X}$ and $\mathfrak{X}^{-}:=\operatorname{Sp}(\mathbb{V}) \cdot e P_{n}$ are also partial flag varieties consisting of all the Lagrangian subspaces. Thus there exist natural embeddings

$$
\mathrm{O}(\mathbb{V}) / P_{n}^{+} \simeq \mathfrak{X}^{+} \hookrightarrow \mathfrak{X}, \quad \operatorname{Sp}(\mathbb{V}) / P_{n}^{-} \simeq \mathfrak{X}^{-} \hookrightarrow \mathfrak{X},
$$


where $P_{n}^{+}=\operatorname{Stab}_{\mathrm{O}(\mathbb{V})}(V)$ and $P_{n}^{-}=\operatorname{Stab}_{\mathrm{Sp}(\mathbb{V})}(V)$ are maximal parabolic subgroups respectively. It is clear $\mathcal{Z}=\mathfrak{X}^{+} \cap \mathfrak{X}^{-}$so that $\mathcal{Z}_{p, q} \simeq \operatorname{Grass}_{p}(V)$ can be regarded as a closed $K$-orbit in both $\mathfrak{X}^{+}$and $\mathfrak{X}^{-}$. Note that

$$
K=\mathrm{GL}(V) \simeq \mathrm{O}(\mathbb{V}) \cap \mathrm{Sp}(\mathbb{V}),
$$

and that $(\mathrm{O}(\mathbb{V}), K)$ and $(\mathrm{Sp}(\mathbb{V}), K)$ are complex symmetric pairs corresponding to real symmetric pairs $\left(\mathrm{O}_{2 n}^{*}, \mathrm{U}_{n}(\mathbb{C})\right)$ and $\left(\mathrm{Sp}_{2 n}(\mathbb{R}), \mathrm{U}_{n}(\mathbb{C})\right)$ respectively.

\section{CASE OF $\mathrm{Sp}_{2 n}(\mathbb{R}) \times \mathrm{O}_{n, n}(\mathbb{R})$}

We consider the standard symmetric bilinear form on $U\left(=U_{1}\right)$ induced by the identity matrix. Then we can define the orthogonal group $\mathrm{O}(U)$ with respect to the symmetric form. By this non-degenerate symmetric form, we can identify $U$ and $U^{*}$, and we put $U_{2}=U^{*}$. Thus it becomes that $H=\mathrm{GL}(U) \times \mathrm{GL}\left(U^{*}\right)$. We put $K^{\prime}:=\mathrm{O}(U) \times \mathrm{O}\left(U^{*}\right) \subset H$. Note that $K^{\prime}$ is the complexification of a maximal compact subgroup $\mathrm{O}_{n}(\mathbb{R}) \times \mathrm{O}_{n}(\mathbb{R})$ of an indefinite orthogonal group $\mathrm{O}_{n, n}(\mathbb{R})$. Similarly, in this section, $K=\mathrm{GL}_{n}(\mathbb{C})$ should be regarded as the complexification of a maximal compact subgroup $\mathrm{U}_{n}(\mathbb{C})$ of $\operatorname{Sp}_{2 n}(\mathbb{R})$, and we use the setting for the dual pair $\left(\operatorname{Sp}_{2 n}(\mathbb{R}), \mathrm{O}_{n, n}(\mathbb{R})\right)$.

Recall the $H \times K$-equivariant resolution of singularities

$$
\widetilde{\mathfrak{N}}_{p, q}=\operatorname{Hom}\left(\mathcal{Q}_{p}, \mathcal{U}\right) \times \operatorname{Hom}\left(\mathcal{U}, \mathcal{T}_{p}\right) \rightarrow \mathfrak{N}_{p, q}
$$

of the irreducible component $\mathfrak{N}_{p, q}$ of the null fiber $(p+q=n)$. Since $H$ acts on the vector bundle $\operatorname{Hom}\left(\mathcal{Q}_{p}, \mathcal{U}\right) \times \operatorname{Hom}\left(\mathcal{U}, \mathcal{T}_{p}\right)$ fiber wise, we can take a categorical quotient by the action of $K^{\prime}=\mathrm{O}(U) \times \mathrm{O}\left(U^{*}\right)$ easily, and get

$$
\operatorname{Hom}\left(\mathcal{Q}_{p}, \mathcal{U}\right) \times \operatorname{Hom}\left(\mathcal{U}, \mathcal{T}_{p}\right) / / K^{\prime} \simeq \operatorname{Sym}\left(\mathcal{Q}_{p}^{*}\right) \times \operatorname{Sym}\left(\mathcal{T}_{p}\right)
$$

where $\operatorname{Sym}(X)$ denotes the symmetric tensor of $X$ in $X \otimes X$. It is a vector bundle over $\operatorname{Grass}_{p}(V) \simeq \mathcal{Z}_{p, q}$ on which $K=\mathrm{GL}(V)$ acts.

Theorem 5.1. Let us denote the conormal bundle of $\mathcal{Z}_{p, q} \subset \mathfrak{X}^{-}$by $T_{\mathcal{Z}_{p, q}}^{*} \mathfrak{X}^{-}$. Then there exists a K-equivariant isomorphism

$$
T_{\mathcal{Z}_{p, q}}^{*} \mathfrak{X}^{-} \simeq \operatorname{Sym}\left(\mathcal{Q}_{p}^{*}\right) \times \operatorname{Sym}\left(\mathcal{T}_{p}\right) \simeq \widetilde{\mathfrak{N}}_{p, q} / / K^{\prime}
$$

Thus the conormal bundle of the closed $K$-orbit $\mathcal{Z}_{p, q}$ in $\mathfrak{X}^{-}$is obtained by taking the categorical quotient of the resolution of singularities $\widetilde{\mathfrak{N}}_{p, q}$ of $\mathfrak{N}_{p, q}$.

Proof. We have to prove $T_{\mathcal{Z}_{p, q}}^{*} \mathfrak{X}^{-} \simeq \operatorname{Sym}\left(\mathcal{Q}_{p}^{*}\right) \times \operatorname{Sym}\left(\mathcal{T}_{p}\right)$. Let us choose a point $\mathbb{L} \in \mathcal{Z}_{p, q}$. Then the tangent space of $\mathfrak{X}^{-}$at $\mathbb{L}$ can be identified with $\operatorname{Sym}\left(\mathbb{L}^{*}\right)$, while the tangent space of $\mathcal{Z}_{p, q}$ at $\mathbb{L}=\mathbb{L}_{V} \oplus \mathbb{L}_{V^{*}}$ should be

$$
\operatorname{Hom}\left(\mathbb{L}_{V}, V / \mathbb{L}_{V}\right) \simeq \mathbb{L}_{V}^{*} \otimes\left(V / \mathbb{L}_{V}\right) \simeq \mathbb{L}_{V}^{*} \otimes \mathbb{L}_{V^{*}}^{*}
$$

(recall that $\left.\mathbb{L}_{V^{*}}=\left(\mathbb{L}_{V}\right)^{\perp}\right)$. Since $\operatorname{Sym}\left(\mathbb{L}^{*}\right) \simeq \operatorname{Sym}\left(\mathbb{L}_{V}^{*}\right) \oplus \operatorname{Sym}\left(\mathbb{L}_{V^{*}}^{*}\right) \oplus \mathbb{L}_{V}^{*} \otimes \mathbb{L}_{V^{*}}^{*}$, we have

$$
T_{\mathbb{L}} \mathfrak{X}^{-} / T_{\mathbb{L}} \mathcal{Z}_{p, q} \simeq \operatorname{Sym}\left(\mathbb{L}^{*}\right) / \operatorname{Hom}\left(\mathbb{L}_{V}, V / \mathbb{L}_{V}\right) \simeq \operatorname{Sym}\left(\mathbb{L}_{V}^{*}\right) \oplus \operatorname{Sym}\left(\mathbb{L}_{V^{*}}^{*}\right)
$$


Thus, the fiber of the conormal bundle $T_{\mathcal{Z}_{p, q}}^{*} \mathfrak{X}^{-}$at $\mathbb{L}$ is $\operatorname{Sym}\left(\mathbb{L}_{V}\right) \oplus \operatorname{Sym}\left(\mathbb{L}_{V^{*}}\right)$.

On the other hand, the fiber of $\mathcal{T}_{p}$ at $\mathbb{L}$ is $\mathbb{L}_{V}$, while that of $\mathcal{Q}_{p}^{*}$ is $\left(V / \mathbb{L}_{V}\right)^{*} \simeq \mathbb{L}_{V^{*}}$. Therefore the fiber of $\operatorname{Sym}\left(\mathcal{Q}_{p}^{*}\right) \times \operatorname{Sym}\left(\mathcal{T}_{p}\right)$ is naturally identified with $\operatorname{Sym}\left(\mathbb{L}_{V}\right) \oplus \operatorname{Sym}\left(\mathbb{L}_{V^{*}}\right)$.

For $p+q=n$, let us consider the nilpotent $K$-orbit $\mathbb{O}_{p, q}$ which is lifted from the trivial $K^{\prime}$-orbit $\{0\}$ in $\mathfrak{p}^{\prime}$. Their shapes are all the same, namely it is $2^{n}$, and their signed Young diagrams are $(+-)^{p}(-+)^{q}$.

Theorem 5.2. The affine quotient $\mathfrak{N}_{p, q} / / K^{\prime}$ is isomorphic to $\overline{\mathbb{O}}_{p, q}$. Moreover, the image of the natural moment map $\mu: T_{\mathcal{Z}_{p, q}}^{*} \mathfrak{X}^{-} \rightarrow \mathfrak{p}$ coincides with $\overline{\mathbb{O}}_{p, q}$, and $\mu$ gives a resolution of singularities of $\overline{\mathbb{O}}_{p, q}$. Thus we have the following commutative diagram.

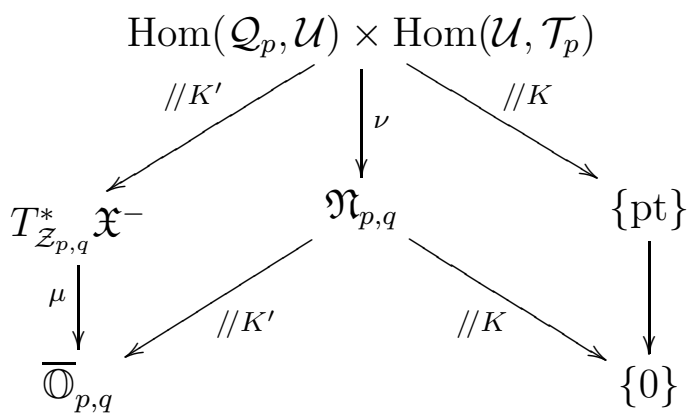

Proof. Let us denote $X=\mathfrak{N}_{p, q} / / K^{\prime}$. Take subspaces $U^{p} \subset U$ and $U^{* q} \subset U^{*}$ of dimension $p$ and $q$ respectively, and assume that the symmetric form restricted to these spaces are non-degenerate. If we put $W^{p, q}=\operatorname{Hom}\left(V, U^{p}\right) \times \operatorname{Hom}\left(U^{q}, V\right)$, then the image of $\mathfrak{N}_{p, q} \cap W^{p, q}$ by the moment map $\varphi$ is $\overline{\mathbb{O}}_{p, q}$ by Theorem 3.2 (see also $\$ 2.3$ ). Thus $X$ contains $\overline{\mathbb{O}}_{p, q}$ as a closed subvariety. On the other hand, from Theorem 1.3, we can calculate

$$
\mathbb{C}[X]=\mathbb{C}\left[\mathfrak{N}_{p, q}\right]^{K^{\prime}} \simeq \bigoplus_{\alpha \in \mathcal{P}_{p}, \beta \in \mathcal{P}_{q}}\left(\rho_{\alpha}^{(n) *} \otimes \rho_{\beta}^{(n)}\right)^{K^{\prime}} \otimes \rho_{\alpha \odot \beta}^{(n)} \simeq \bigoplus_{\alpha \in \mathcal{P}_{p}, \beta \in \mathcal{P}_{q}} \rho_{2 \alpha \odot 2 \beta}^{(n)}
$$

as a $K$-module. The same calculation leads the conclusion that it is isomorphic to $\mathbb{C}\left[\overline{\mathbb{O}}_{p, q}\right]$ (see also [Nis00]). Therefore we must have $X=\overline{\mathbb{O}}_{p, q}$.

Let us prove that $\mu$ is a resolution of $\overline{\mathbb{O}}_{p, q}$. Since the conormal bundle is a bundle over a projective variety $\mathcal{Z}_{p, q}, \mu$ is proper. Take $x \in \mathbb{O}_{p, q}$. Then it is easy to see that the fiber $\varphi^{-1}(x)$ is a single $K^{\prime}$-orbit, which is contained in $\mathfrak{N}_{p, q}^{\circ}$. Since $\nu$ restricted to $\nu^{-1}\left(\mathfrak{N}_{p, q}^{\circ}\right) \rightarrow \mathfrak{N}_{p, q}^{\circ}$ is isomorphism, the fiber $\mu^{-1}(x)$ consists of a single point. Thus $\mu$ is birational. The closure $\overline{\mathbb{O}}_{p, q}$ contains a nilpotent orbit $\mathbb{O}_{s, t}(s \leq p, t \leq q)$, and no other orbit can adherent to $\mathbb{O}_{p, q}$. For $y \in \mathbb{O}_{s, t}$, it is easy to see that $\mu^{-1}(y)$ has positive dimension. 
6. CASE OF $\mathrm{O}_{2 n}^{*} \times \mathrm{Sp}_{n, n}$

Let us assume that $n$ is even. Then the results in $\$ 5$ have analogue for the pair $\mathrm{O}_{2 n}^{*} \times$ $\mathrm{Sp}_{n, n}$. We only state the results here.

We consider a symplectic form on $U\left(=U_{1}\right)$, and define the symplectic group $\operatorname{Sp}(U)$ with respect to the form. By this non-degenerate symplectic form, we can identify $U$ and $U^{*}$, and we put $U_{2}=U^{*}$. Thus it becomes that $H=\operatorname{GL}(U) \times \operatorname{GL}\left(U^{*}\right)$ and we put $K^{\prime}:=\operatorname{Sp}(U) \times \operatorname{Sp}\left(U^{*}\right) \subset H$. Note that $K^{\prime}$ is the complexification of a maximal compact subgroup $\mathrm{USp}_{n} \times \mathrm{USp}_{n}$ of the indefinite symplectic group $\mathrm{Sp}_{n, n}$ over Hamiltonian numbers, where $\mathrm{USp}_{n}$ denotes the unitary symplectic group of size $n$ (the rank is $n / 2$ ). Similarly, in this section, $K=\mathrm{GL}_{n}(\mathbb{C})$ should be regarded as the complexification of a maximal compact subgroup $\mathrm{U}_{n}(\mathbb{C})$ of $\mathrm{O}_{2 n}^{*}$. and we are working on the dual pair $\left(\mathrm{O}_{2 n}^{*}, \mathrm{Sp}_{n, n}\right)$.

Theorem 6.1. Let us denote the conormal bundle of $\mathcal{Z}_{p, q} \subset \mathfrak{X}^{+}$by $T_{\mathcal{Z}_{p, q}}^{*} \mathfrak{X}^{+}$. Then there exists a K-equivariant isomorphism

$$
T_{\mathcal{Z}_{p, q}}^{*} \mathfrak{X}^{+} \simeq \operatorname{Alt}\left(\mathcal{Q}_{p}^{*}\right) \times \operatorname{Alt}\left(\mathcal{T}_{p}\right) \simeq \widetilde{\mathfrak{N}}_{p, q} / / K^{\prime},
$$

where Alt $(X)$ denotes the alternating tensors over $X$ of the second degree. Thus the conormal bundle of the closed $K$-orbit $\mathcal{Z}_{p, q}$ in $\mathfrak{X}^{+}$is obtained by taking the categorical quotient of the resolution of singularities $\widetilde{\mathfrak{N}}_{p, q}$ of $\mathfrak{N}_{p, q}$.

Let us consider nonnegative even integers $p$ and $q$ such that $p+q=n$. Then there is a nilpotent $K$-orbit $\mathbb{O}_{p, q}$ which is lifted from the trivial $K^{\prime}$-orbit $\{0\}$ in $\mathfrak{p}^{\prime}$. Their shapes are all the same, namely it is $2^{n}$, and their signed Young diagrams are $(+-)^{p}(-+)^{q}$.

Theorem 6.2. Assume that $p, q$ are even and satisfy $p+q=n$. Then the affine quotient $\mathfrak{N}_{p, q} / / K^{\prime}$ is isomorphic to $\overline{\mathbb{O}}_{p, q}$. Moreover, the image of the moment map $\mu: T_{\mathcal{Z}_{p, q}}^{*} \mathfrak{X}^{+} \rightarrow \mathfrak{p}$ coincides with $\overline{\mathbb{O}}_{p, q}$, and $\mu$ gives a resolution of singularities of $\overline{\mathbb{O}}_{p, q}$. Thus we have the following commutative diagram.

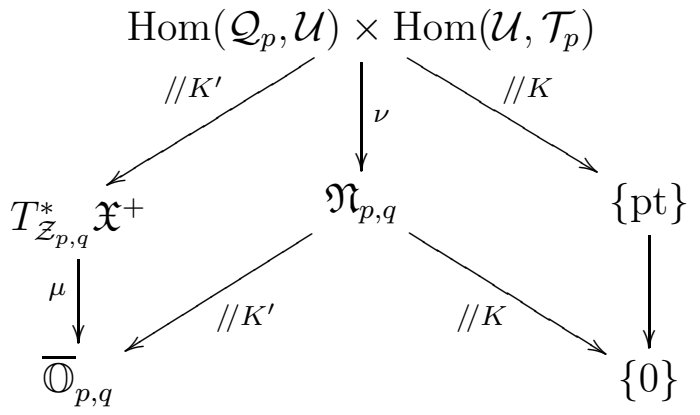

Let us take even integers $p$ and $q$ such that $p+q=n$ as above. Then $\mathfrak{N}_{p+1, q-1} / / K^{\prime}=$ $\overline{\mathbb{O}}_{p, q-2}$ which is adherent to $\mathbb{O}_{p, q}$. Such kind of degeneration of the categorical quotient makes the situation more complicated than the case of dual pair $\left(\operatorname{Sp}_{2 n}(\mathbb{R}), \mathrm{O}_{n, n}(\mathbb{R})\right)$. 


\section{REFERENCES}

[Bry93] Ranee Kathryn Brylinski, Matrix concomitants with the mixed tensor model, Adv. Math. 100 (1993), no. 1, 28-52.

[DKP02] Andrzej Daszkiewicz, Witold Kraśkiewicz, and Tomasz Przebinda, Dual pairs and KostantSekiguchi correspondence. I, J. Algebra 250 (2002), no. 2, 408-426.

[DKP05] Andrzej Daszkiewicz, Witold Kraśkiewicz, and Tomasz Przebinda, Dual pairs and KostantSekiguchi correspondence. II. Classification of nilpotent elements, Cent. Eur. J. Math. 3 (2005), no. 3, 430-474 (electronic).

[GW98] Roe Goodman and Nolan R. Wallach, Representations and invariants of the classical groups, Encyclopedia of Mathematics and its Applications, vol. 68, Cambridge University Press, Cambridge, 1998.

[Har77] Robin Hartshorne, Algebraic geometry, Springer-Verlag, New York, 1977, Graduate Texts in Mathematics, No. 52.

[How85] Roger Howe, Dual pairs in physics: harmonic oscillators, photons, electrons, and singletons, Applications of group theory in physics and mathematical physics (Chicago, 1982), Lectures in Appl. Math., vol. 21, Amer. Math. Soc., Providence, RI, 1985, pp. 179-207.

[How89] Roger Howe, Remarks on classical invariant theory, Trans. Amer. Math. Soc. 313 (1989), no. 2, 539-570.

[How95] Roger Howe, Perspectives on invariant theory: Schur duality, multiplicity-free actions and beyond, The Schur lectures (1992) (Tel Aviv), Israel Math. Conf. Proc., vol. 8, Bar-Ilan Univ., Ramat Gan, 1995, pp. 1-182.

[KV78] M. Kashiwara and M. Vergne, On the Segal-Shale-Weil representations and harmonic polynomials, Invent. Math. 44 (1978), no. 1, 1-47.

[Nis00] Kyo Nishiyama, Theta lifting of two-step nilpotent orbits for the pair $\mathrm{O}(p, q) \times \operatorname{Sp}(2 n, \mathbb{R})$, Infinite dimensional harmonic analysis (Kyoto, 1999), Gräbner, Altendorf, 2000, pp. 278-289.

[Nis05] Kyo Nishiyama, A note on affine quotients and equivariant double fibrations, Infinite dimensional harmonic analysis III, World Sci. Publ., Hackensack, NJ, 2005, pp. 197-212.

[NOZ06] Kyo Nishiyama, Hiroyuki Ochiai, and Chen-Bo Zhu, Theta lifting of nilpotent orbits for symmetric pairs, Trans. Amer. Math. Soc. 358 (2006), no. 6, 2713-2734 (electronic).

Department of Mathematics, Graduate School of Science, Kyoto University, Sakyo, KYOTO 606-8502, JAPAN

E-mail address: kyo@math.kyoto-u.ac.jp 